\title{
NONEXISTENCE OF CUBIC DDI GRAPHS OF ORDER 16 WITH DIAMETERS 4, 5, 6
}

\section{Medha Itagi Huilgol and M. Rajeshwari}

Department of Mathematics

Bangalore University

Central College Campus

Bangalore - 560 001, India

e-mail: medha@bub.ernet.in

rajeswarim@acharya.ac.in

\begin{abstract}
The eccentricity $e(u)$ of a vertex $u$ is the maximum distance of $u$ to any other vertex of $G$. The distance degree sequence (dds) of a vertex $v$ in a graph $G=(V, E)$ is a list of the number of vertices at distance $1,2, \ldots, e(u)$ in that order, where $e(u)$ denotes the eccentricity of $v$ in $G$. Thus, the sequence $\left(d_{i_{0}}, d_{i_{1}}, d_{i_{2}}, \ldots, d_{i_{j}}, \ldots\right)$ is the distance degree sequence of the vertex $v_{i}$ in $G$, where $d_{i_{j}}$ denotes the number of vertices at distance $j$ from $v_{i}$. A graph is distance degree regular (DDR) graph if all the vertices have the same distance degree sequence. A graph is distance degree injective (DDI) graph if no two vertices have the same distance degree sequence. In this paper, we prove that there does not exist cubic DDI graphs of order 16 with diameters 4, 5, 6 .
\end{abstract}

Received: December 16, 2014; Accepted: March 12, 2015

2010 Mathematics Subject Classification: 05C12.

Keywords and phrases: eccentric vertex, distance degree sequence, DDR graph, DDI graph. Communicated by K. K. Azad 